\title{
Molecular Abundances in AGB Circumstellar Envelopes
}

\author{
Hans Olofsson \\ Stockholm Observatory, AlbaNova, SE-10691 Stockholm, Sweden \\ email: hans@astro.su.se
}

\begin{abstract}
In this review the present status of molecules in circumstellar envelopes of AGB stars is presented. Emphasis is put on the determination of abundances, and estimates of their uncertainties, from an observational point of view. Despite an impressive number of circumstellar species detected, about 60 , there remains much work before general conclusions can be drawn. In particular, sophisticated radiative transfer modelling of circumstellar line emission must be done. This requires a detailed knowledge of the stellar and circumstellar properties, as well as basic molecular physics/chemistry data.
\end{abstract}

Keywords. stars: AGB and post-AGB — stars: abundances — stars: mass loss — stars: circumstellar matter - radiative transfer

\section{Introduction}

The circumstellar envelopes (CSEs) of Asymptotic Giant Branch (AGB) stars, formed through extensive stellar mass loss, contain gas (primarily in the form of molecules) and dust. The molecules are formed through a sequence of processes. The high-density, hightemperature LTE chemistry in the stellar atmosphere produces the parent molecules. The result is strongly determined by the $\mathrm{C} / \mathrm{O}$ ratio in the atmosphere. The mass-loss mechanism expels these molecules through the dynamical atmospheres of the pulsating AGB star. Presumably, this activates a shock chemistry that partly changes the chemical composition of the outflowing gas. The formation of bulk particles, dust grains, also takes place in the upper stellar atmosphere. This may decrease some molecular abundances because of grain adsorption, while new molecules are produced through grain surface chemistry. Eventually, the dispersion of the gas/dust CSE leads to the destruction of molecules by interstellar UV photons. In this way new molecules are formed as photodissociation products, and a photo-induced chemistry is initiated where these play a crucial role. Even if this sequence of processes may lead to some surprising results, the molecular setup of a CSE is primarily determined by the stellar C/O ratio (Millar 2003). The general elemental composition of the star shows up only in the finer details.

The ultimate goal is to understand the stellar/circumstellar chemistry on the AGB (and beyond), and from this derive important results relevant to stellar evolution as well as galactic chemical evolution. The relative simplicity of the AGB circumstellar environment lends the hope that various chemical processes can be studied in great detail. In addition, through the radial structure produced by the outflowing gas and dust, there is also the possibility of understanding 'time-resolved' chemistry.

\section{The AGB Circumstellar Environment}

The circumstellar envelope around an AGB star provides, at least to a first approximation, a rather well-defined environment in which physical and chemical processes can 
be studied. Overall, they are spherically symmetric, and expand with a constant velocity. A constant mass-loss rate (at least over the time scale probed by molecular emissions, $\leqslant 10^{4} \mathrm{yr}$ ) assures that the densities scale as $r^{-2}$. The properties of the radiation fields, due to the aging red giant and circumstellar dust, are relatively well characterized. A unique property of AGB CSEs is that they provide two distinctly different chemical environments, those that are $\mathrm{O}$-rich $(\mathrm{C} / \mathrm{O}<1)$ and those that are $\mathrm{C}$-rich $(\mathrm{C} / \mathrm{O}>1)$. Finally, regular luminosity variations of the central star and the expansion of the CSE allow temporal chemical evolution studies.

Basic to all circumstellar abundance estimates is a good circumstellar model, which is normally obtained in two ways. Radiative transfer modelling of $\mathrm{CO}$ radio line emission combined with a solution of the energy-balance equation provides the mass-loss rate (assuming a CO abundance), the terminal expansion velocity, and the kinetic temperature law (Groenewegen 1994; Schöier \& Olofsson 2001). Radiative transfer modelling of the spectral energy distribution combined with a dust-driven-wind model provide an estimate of the mass-loss rate, the dust mass-loss rate, the velocity law, and the dust radiation field. In general, the mass-loss rate, which enters the abundance through the $\mathrm{H}_{2}$ density, of an individual object has an uncertainty of at least a factor of three.

\section{Circumstellar Molecules}

There are presently 63 molecular species detected in AGB CSEs, Table 1, and a significant number of them are unique to the circumstellar medium (when compared to the interstellar medium). About $80 \%$ are detected at radio wavelengths, and the rest (except $\mathrm{C}_{2}$ ) are detected at IR wavelengths. This is impressive considering that AGB CSEs are low-mass objects.

However, the situation is far from satisfactory. About half of the molecules have been detected in only one object, IRC +10216 , and an additional 10 in less than 10 objects. Measured brightness distributions are essentially limited to a dozen species, in a single line each, observed towards IRC +10216 (with the exception of CO). The CSE of IRC+10216 has turned out to be a gold mine, since this object is probably the nearbest C-star $(\approx 120 \mathrm{pc})$, and it happens to have a very high mass-loss rate $\left(\approx 2 \times 10^{-5} \mathrm{M}_{\odot} \mathrm{yr}^{-1}\right)$. If it is also representative of a C-rich CSE remains to be determined.

In the C-rich CSE of IRC+10216 a large number of relatively complicated molecules exists, mainly as the result of an efficient C-based circumstellar chemistry. Here we find sequences of long carbon chains: the cyanopolyynes $\mathrm{HC}_{n} \mathrm{~N}$, the hydrocarbons $\mathrm{C}_{n} \mathrm{H}$ (Cernicharo \& Guélin 1996), and the carbenes $\mathrm{H}_{2} \mathrm{C}_{n}$ (Cernicharo et al. 1991). Nitrogencarbon chains $\mathrm{C}_{n} \mathrm{~N}$ (Guélin et al. 1998), silicon-carbon chains $\mathrm{C}_{n} \mathrm{Si}$ (Ohishi et al. 1989), and sulphur-carbon chains $\mathrm{C}_{n} \mathrm{~S}$ (Cernicharo et al. 1987) are also present. Simple ring molecules $\left(\mathrm{SiC}_{2}\right.$, cyclic $\mathrm{C}_{3} \mathrm{H}_{2}$, and $\mathrm{SiC}_{3}$ ) have been detected (Apponi et al. 1999) as well, but branched molecules are notably rare. On the spectacular side, we have the 'metal' species $\mathrm{NaCl}, \mathrm{AlCl}, \mathrm{AlF}, \mathrm{KCl}, \mathrm{MgNC}$, etc. (Cernicharo \& Guélin 1987; Ziurys et al. 2002). Notably, only one ion, $\mathrm{HCO}^{+}$, has been detected. Most likely, even more complicated species exist in C-rich AGB CSEs. Their detections may come through sub-mm and far-IR observations of ro-vibrational lines of various bending and flopping modes.

The O-CSEs are apparently less rich in molecules, certainly an effect of the lack of the chemically potent carbon atoms. However, the situation may have been slightly different had an M-star equivalent in mass-loss rate and proximity to IRC+10216 existed. Here $\mathrm{OH}, \mathrm{H}_{2} \mathrm{O}$, and $\mathrm{SiO}$ dominate (through their strong maser lines), but also S-bearing species, e.g., $\mathrm{SO}, \mathrm{SO}_{2}$, and $\mathrm{H}_{2} \mathrm{~S}$, are frequently found (Omont et al. 1993). Quite unexpectedly, detections of C-bearing species, $\mathrm{HCN}$, CS, and $\mathrm{CN}$, have been made (Olofsson 
Table 1. Molecules detected in AGB CSEs. The (rough) number of sources detected in each species is given $(\Sigma)$, as well as abundances w.r.t. $\mathrm{H}_{2}\left(\mathrm{O}: \mathrm{C} / \mathrm{O}<1 ; \mathrm{C}: \mathrm{C} / \mathrm{O}>1 ; k(l)=k \times 10^{l}\right)$

\begin{tabular}{|c|c|c|c|c|c|c|c|c|}
\hline & \multirow[t]{2}{*}{ Molecule } & \multirow[t]{2}{*}{$\Sigma$} & \multicolumn{2}{|c|}{ Chem. } & \multirow[t]{2}{*}{ Molecule } & \multirow[t]{2}{*}{$\Sigma$} & \multicolumn{2}{|c|}{ Chem. } \\
\hline & & & $\mathrm{O}$ & $\mathrm{C}$ & & & $\mathrm{O}$ & $\mathrm{C}$ \\
\hline 2-atoms & $\begin{array}{l}\mathrm{AlCl} \\
\mathrm{AlF} \\
\mathrm{C}_{2} \\
\mathrm{CO} \\
\mathrm{CN} \\
\mathrm{CP} \\
\mathrm{CS} \\
\mathrm{KCl}\end{array}$ & $\begin{array}{r}1 \\
1 \\
1 \\
600 \\
40 \\
1 \\
35 \\
1\end{array}$ & $\begin{array}{l}5(-4) \\
2(-7) \\
1(-7)\end{array}$ & $\begin{array}{l}1(-7) \\
4(-8) \\
2(-6) \\
1(-3) \\
5(-6) \\
2(-8) \\
1(-6) \\
2(-9)\end{array}$ & $\begin{array}{l}\mathrm{NaCl} \\
\mathrm{OH} \\
\mathrm{PN} \\
\mathrm{SiC} \\
\mathrm{SiN} \\
\mathrm{SiO} \\
\mathrm{SiS} \\
\mathrm{SO}\end{array}$ & $\begin{array}{r}1 \\
2000 \\
1 \\
2 \\
1 \\
500 \\
20 \\
20\end{array}$ & $\begin{array}{l}5(-6) \\
7(-7) \\
2(-6)\end{array}$ & $\begin{array}{l}1(-9) \\
4(-8) \\
? \\
4(-8) \\
2(-8) \\
1(-7) \\
2(-6)\end{array}$ \\
\hline 3-atoms & $\begin{array}{l}\mathrm{AlNC} \\
\mathrm{C}_{3} \\
\mathrm{C}_{2} \mathrm{H} \\
\mathrm{C}_{2} \mathrm{~S} \\
\mathrm{CO}_{2} \\
\mathrm{HCN} \\
\mathrm{H}_{2} \mathrm{O} \\
\mathrm{H}_{2} \mathrm{~S}\end{array}$ & $\begin{array}{r}1 \\
1 \\
20 \\
5 \\
15 \\
120 \\
300 \\
20\end{array}$ & $\begin{array}{l}3(-7) \\
4(-6) \\
3(-4) \\
1(-5)\end{array}$ & $\begin{array}{l}1(-9) \\
1(-6) \\
4(-6) \\
1(-6) \\
2(-5) \\
1(-6)\end{array}$ & $\begin{array}{l}\mathrm{HNC} \\
\mathrm{MgCN} \\
\mathrm{MgNC} \\
\mathrm{NaCN} \\
\mathrm{SiC}_{2} \\
\mathrm{SiCN} \\
\mathrm{SiNC} \\
\mathrm{SO}_{2}\end{array}$ & $\begin{array}{r}15 \\
1 \\
1 \\
1 \\
5 \\
1 \\
1 \\
15\end{array}$ & $1(-7)$ & $\begin{array}{l}1(-7) \\
1(-9) \\
2(-8) \\
2(-8) \\
3(-7) \\
4(-9) \\
4(-9)\end{array}$ \\
\hline 4-atoms & $\begin{array}{l}\ell-\mathrm{C}_{3} \mathrm{H} \\
\mathrm{C}_{3} \mathrm{~N} \\
\mathrm{C}_{3} \mathrm{~S} \\
\mathrm{C}_{2} \mathrm{H}_{2}\end{array}$ & $\begin{array}{l}2 \\
5 \\
1 \\
7\end{array}$ & & $\begin{array}{l}4(-8) \\
3(-7) \\
3(-8) \\
5(-5)\end{array}$ & $\begin{array}{l}\mathrm{HC}_{2} \mathrm{~N} \\
\mathrm{H}_{2} \mathrm{CO} \\
\mathrm{NH}_{3} \\
\mathrm{SiC}_{3}\end{array}$ & $\begin{array}{l}1 \\
1 \\
5 \\
1\end{array}$ & $4(-6)$ & $\begin{array}{l}8(-9) \\
1(-8) \\
1(-7) \\
3(-9)\end{array}$ \\
\hline 5-atoms & $\begin{array}{l}\mathrm{C}_{5} \\
\mathrm{C}_{4} \mathrm{H} \\
\mathrm{C}_{4} \mathrm{Si} \\
\mathrm{c}-\mathrm{C}_{3} \mathrm{H}_{2} \\
\mathrm{CH}_{4}\end{array}$ & $\begin{array}{l}1 \\
5 \\
1 \\
5 \\
1\end{array}$ & & $\begin{array}{l}1(-7) \\
3(-6) \\
3(-9) \\
3(-8) \\
4(-6)\end{array}$ & $\begin{array}{l}\mathrm{HC}_{3} \mathrm{~N} \\
\mathrm{HC}_{2} \mathrm{NC} \\
\mathrm{H}_{2} \mathrm{C}_{3} \\
\mathrm{SiH}_{4}\end{array}$ & $\begin{array}{r}10 \\
1 \\
1 \\
1\end{array}$ & & $\begin{array}{l}1(-6) \\
2(-9) \\
2(-9) \\
2(-7)\end{array}$ \\
\hline 6-atoms & $\begin{array}{l}\mathrm{C}_{5} \mathrm{H} \\
\mathrm{C}_{5} \mathrm{~N} \\
\mathrm{C}_{2} \mathrm{H}_{4}\end{array}$ & $\begin{array}{l}1 \\
1 \\
1\end{array}$ & & $\begin{array}{l}6(-8) \\
9(-9) \\
1(-8)\end{array}$ & $\begin{array}{l}\mathrm{CH}_{3} \mathrm{CN} \\
\mathrm{HC}_{4} \mathrm{~N} \\
\mathrm{H}_{2} \mathrm{C}_{4}\end{array}$ & $\begin{array}{l}5 \\
1 \\
1\end{array}$ & & $\begin{array}{l}3(-9) \\
1(-9) \\
5(-9)\end{array}$ \\
\hline$\geqslant 7$-atoms & $\begin{array}{l}\mathrm{C}_{6} \mathrm{H} \\
\mathrm{C}_{7} \mathrm{H} \\
\mathrm{C}_{8} \mathrm{H} \\
\mathrm{HC}_{5} \mathrm{~N}\end{array}$ & $\begin{array}{l}1 \\
1 \\
1 \\
5\end{array}$ & & $\begin{array}{l}8(-8) \\
3(-9) \\
1(-8) \\
2(-7)\end{array}$ & $\begin{array}{l}\mathrm{HC}_{7} \mathrm{~N} \\
\mathrm{HC}_{9} \mathrm{~N} \\
\mathrm{H}_{2} \mathrm{C}_{6}\end{array}$ & $\begin{array}{l}2 \\
1 \\
1\end{array}$ & & $\begin{array}{c}4(-8) \\
1(-8) \\
?\end{array}$ \\
\hline Ions: & $\mathrm{HCO}^{+}$ & 2 & & $1(-9)$ & & & & \\
\hline
\end{tabular}

et al. 1991; Bujarrabal et al. 1994), showing that there exists a source of free carbon atoms that drive a carbon chemistry in these CSEs.

The increasing ultraviolet flux and the presence of shocks in post-AGB objects should have a noticeable effect on the chemistry (Woods et al. 2003a). This is verified by the detections of polyacetylenes in the C-rich proto-PNe AFGL618 and AFGL2688, and methylpolyynes and the benzene ring in AFGL618 (Cernicharo et al. 2001a; Cernicharo et al. 2001b). A number of ionic species have been detected in the CSE around a young PN, NGC7027 (Latter et al. 1993). In addition, abundant molecular hydrogen is readily detectable in PNe of the bipolar type (Cox et al. 2002). The molecular species detected only in post-AGB CSEs are $\mathrm{CH}, \mathrm{CH}^{+}, \mathrm{CO}^{+}, \mathrm{H}_{2}, \mathrm{~N}_{2} \mathrm{H}^{+}, \mathrm{OCS}, \mathrm{HC}_{4} \mathrm{H}, \mathrm{HC}_{6} \mathrm{H}, \mathrm{CH}_{3} \mathrm{C}_{2} \mathrm{H}$, $\mathrm{CH}_{3} \mathrm{C}_{4} \mathrm{H}$, and $\mathrm{C}_{6} \mathrm{H}_{6}$. 


\section{Abundance Estimate Methods}

\subsection{Single Line}

Single-line estimates are commonly used for (simpler) species where few lines have been observed. This works only in the optically thin regime, and requires assumptions about a Boltzmann population distribution at an assumed temperature, as well as an assumption of the size of the emitting region (since this is normally not measured). This leads to order-of-magnitude estimates at best, but it is important to check that the assumption of optically thin emission is fulfilled. It should be noted that even for CO, the lower rotational transitions are very far from thermally excited at low mass-loss rates; in fact, they are inverted over substantial regions of the CSE.

\subsection{Rotational Temperature Diagram}

In particular, heavier molecules and asymmetric tops allow the measurement of line intensities from a large number of transitions (preferably sampling a large range in excitation energies). This can be used in a rotation-temperature diagram to estimate both the excitation temperature and the column density, and should, in principle, lead to an abundance with a (often significantly) higher accuracy than that obtained from a single line. However, the lines must be optically thin, and originate in the same region (its size is often assumed). The latter may not be true in an AGB CSE where the stellar radiation intensity and kinetic temperature gradients are rather steep. Yet, when comparing abundances for a single source this method is expected to provide fairly reliable results.

\subsection{Radiative Transfer Modelling}

Detailed radiative transfer modelling, including a chemical model for the species in question, is still rarely used. There are a number of reasons for this, e.g., the lack of reliable collisional cross sections (however, see Schöier et al. 2005), and a complicated radiative transfer where the details of the radiation field or the molecular energy level structure play an important role. Interestingly, a sophisticated model often leads to abundance estimates with rather large uncertainties.

\section{Abundance Estimates}

The majority of the abundance (with respect to $\mathrm{H}_{2}$ ) estimates given in Table 1 are either based on single lines or using the rotation-temperature-diagram method on multiline data. Only in very few cases have more sophisticated radiative transfer analyses been used. Therefore, the given abundances are in general order-of-magnitude estimates. In addition, for some species the abundance is an average obtained from estimates for a number of sources. We proceed to look at this in more detail.

\subsection{An Individual Source, IRC+10216}

The heavier (often linear) carbon species detected at radio wavelengths are particularly suitable for a rotation-temperature-diagram analysis. This has been used for the IRC+10216 CSE where broad band spectral scans provide the data (e.g., Cernicharo et al. 2000). As an example Figure 1 shows a comparison of a number of carbon-chain species abundances. Although the absolute scale is uncertain, the relative abundances are expected to be rather accurate. The immediate conclusion is that the abundance decrease with increasing 'complexity' is moderate. For the cyanopolyynes, $\mathrm{HC}_{2 n+1} \mathrm{~N}$, the abundance decreases by about a factor of four for each step in the sequence $n=1$ to 4 . The same applies to the related species $\mathrm{C}_{2 n} \mathrm{H}$ when $n$ goes from 1 to 4 . Note also that the 


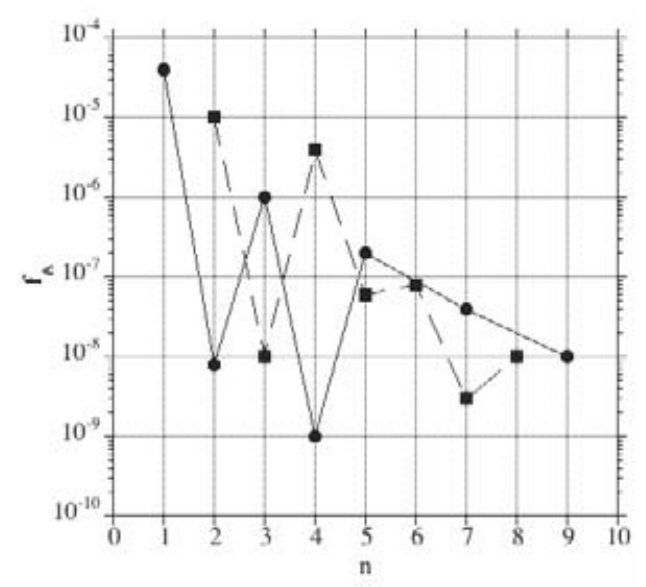

Figure 1. Abundances (with respect to $\mathrm{H}_{2}$ ) of carbon-chain species, $\mathrm{HC}_{n} \mathrm{~N}$ (circles) and $\mathrm{C}_{n} \mathrm{H}$ (squares), in the CSE of the C-star IRC+10216.

$\mathrm{HC}_{2 n} \mathrm{~N}$ and $\mathrm{C}_{2 n+1} \mathrm{H}$ species are considerably less abundant. In addition, ratios involving isomers, e.g., $\mathrm{HC}_{2} \mathrm{NC} / \mathrm{HC}_{3} \mathrm{~N} \approx 0.003$, and branched species, e.g., $\mathrm{CH}_{3} \mathrm{CN} / \mathrm{HCN} \approx 0.0003$, provide further constraints on the chemical models.

Interesting results appear when comparisons with young post-AGB objects are done. Pardo et al. (2005) found that the $\mathrm{HCN} / \mathrm{HC}_{3} \mathrm{~N}$ abundance ratio is at least an order of magnitude lower in the C-rich proto-PN AFGL618 than in IRC+10216, while the decrease in abundance beyond $\mathrm{HC}_{3} \mathrm{~N}$ is the same. This suggests a very efficient transformation of $\mathrm{HCN}$ into longer cyanopolyyne chains. Likewise, polyacetylenes are abundant in AFGL618 $\left(\mathrm{C}_{4} \mathrm{H}_{2}\right.$ and $\mathrm{C}_{6} \mathrm{H}_{2}$ are only a factor of a few less abundant than $\left.\mathrm{C}_{2} \mathrm{H}_{2}\right)$, while they are not detected in IRC+10216 (Cernicharo et al. 2001b). The increasing UV flux and shocks produce efficient factories of organic molecules in young post-AGB objects.

Another interesting, recent development concerns O-bearing molecules in C-rich CSEs. Surprisingly, $\mathrm{H}_{2} \mathrm{O}$ was discovered towards IRC+10216 (Melnick et al. 2001), and a destruction of icy Kuiper-belt-like objects was suggested as an explanation. This stimulated further searches leading to the successful detections of $\mathrm{OH}$ (Ford et al. 2003) and $\mathrm{H}_{2} \mathrm{CO}$ (Ford et al. 2004), but a low upper limit to $\mathrm{CH}_{3} \mathrm{OH}$ (Ford et al. 2004). Further observations are required before the chemical origin is firmly established.

It should be emphasized that whether these are generally applicable results we do not know, since data of these quality exist only towards IRC +10216 .

\subsection{Comparison Between Sources}

Very few studies comparing molecular abundances in different sources have been made. Woods et al. (2003b) observed the same molecular species, 15 in total, in the CSEs of seven high-mass-loss-rate C-stars with similar characteristics (including IRC+10216). Using the single-line method (although crude rotation-temperature estimates are obtained for a few species), and theoretical estimates of the photodissociation radii, they concluded that in five sources (including IRC+10216) the abundances vary between the sources by no more than a factor of five (i.e., within the uncertainties). Two sources, CIT6 and, in particular, IRAS15194-5115, show a somewhat different chemical composition, but this remains to be confirmed by more sophisticated modelling.

A notable exception to the general agreement is $\mathrm{SiO}$ which shows a variation of more than an order of magnitude between the sources, and the abundance is, on average, 




Figure 2. Circumstellar $\mathrm{SiO}$ abundances as a function of stellar mass-loss rate for sample of stars: M-stars (squares) and C-stars (circles). The solid line gives the maximum stellar photospheric abundance for solar abundances, and the dotted line the result of a 'simple' grain condensation model assuming an M-star photospheric equilibrium $\mathrm{SiO}$ abundance of $5 \times 10^{-5}$. The C-star photospheric equilibrium $\mathrm{SiO}$ abundance is about $5 \times 10^{-8}$.

more than $10 \times$ higher than predicted from stellar atmosphere chemistry. This result is confirmed by radiative transfer modelling, see $\S 5.3$.

\subsection{Radiative Transfer Modelling Results}

The excitation conditions in CSEs are, for most molecular species, far from LTE, and the effects of non-local radiative transfer may be substantial (not the least in the case of the stellar radiation field). Thus, collisional cross sections and the details of the radiation fields are often crucial.

$\mathrm{CO}$ is probably the species with the most accurate collisional cross sections, and, in addition, the sensitivity to the radiation fields is limited. However, the abundance of CO is often assumed and serves as an estimator of the $\mathrm{H}_{2}$ density, i.e., the stellar mass-loss rate (Schöier \& Olofsson 2001; Olofsson et al. 2002). This assumption is probably not so $\mathrm{bad}$, since the stability of $\mathrm{CO}$ suggests that it is the $\mathrm{C}$ and $\mathrm{O}$ content that determines the $\mathrm{CO}$ abundance in O- and C-CSEs, respectively, but it leads to a basic uncertainty in other abundance estimates.

The first more detailed study of abundances in a larger sample of sources was performed by González Delgado et al. (2003), who examined SiO in about 40 M-stars. This is a more complicated problem than the radiative transfer modelling of CO: the dependence on radiative excitation is stronger, and the chemistry is expected to be more complex (due to grain formation effects as well as photodissociation). González Delgado et al. (2003) used multi-line data to derive the size of the emitting region, and obtained reasonable fits to both brightness sizes measured by interferometers and theoretical estimates from photodissociation models, as well as the $\mathrm{SiO}$ abundance. Schöier \& Olofsson (2005) used the same method to estimate the SiO abundance in a sample of 17 C-rich CSEs. The $\mathrm{SiO}$ abundance estimates for both samples are given as a function of stellar mass-loss rate in Figure 2. There are some striking results. For the M-stars the circumstellar abundance is much lower than that expected from photospheric equilibrium chemistry $\left(5 \times 10^{-5}\right)$, on average by an order of magnitude, in many cases by two orders of magnitude. For the C-stars the circumstellar abundance is much higher than that expected from 


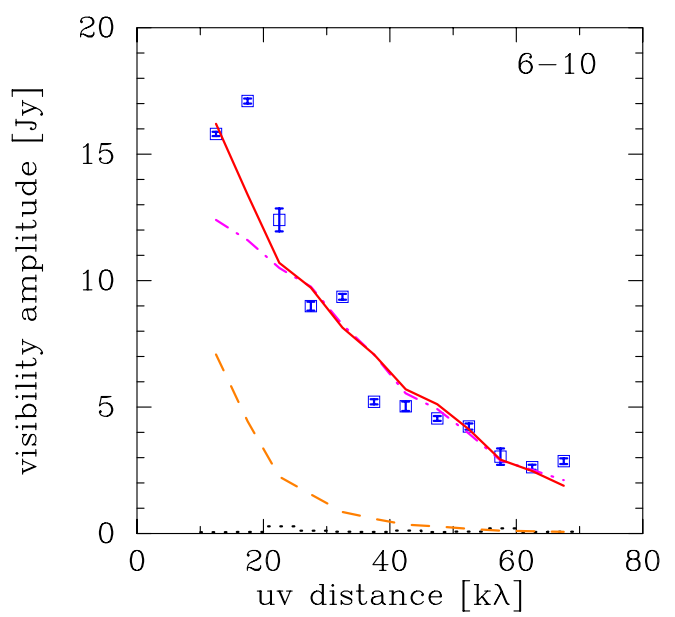

Figure 3. A radiative transfer model fit to the $\mathrm{SiO}(v=0, J=2-1)$ uv data obtained towards the M-star R Dor. The solid line gives the best fit model, which is based on a two-component $\mathrm{SiO}$ abundance distribution (see text). The dashed-dot line gives the result for only an inner high-abundance component, and the dashed line the best-fit model using only single-dish data.

photospheric equilibrium chemistry $\left(5 \times 10^{-8}\right)$, on average by two orders of magnitude. In addition, there is a trend of decreasing circumstellar abundance with increasing massloss rate, probably due to a trend of increasing $\mathrm{SiO}$ depletion onto grains with increasing circumstellar density.

Recent interferometric results show that a more complicated $\mathrm{SiO}$ abundance distribution is required to explain the data (as is also expected due to grain formation and photodissociation). In the case of the semi-regular M-star R Dor Schöier et al. (2004) found that an inner component of high $\mathrm{SiO}$ abundance (at the level expected from stellar atmosphere chemistry), and an outer, extended component of low $\mathrm{SiO}$ abundance (as expected from $\mathrm{SiO}$ depletion onto grains), are required to fit the data, Figure 3.

The final example concerns circumstellar $\mathrm{H}_{2} \mathrm{O}$. Water has been known for decades through the detections of $\mathrm{H}_{2} \mathrm{O}$ (and $\mathrm{OH}$ ) masers. More data were provided by ISO, where the SWS and LWS instruments delivered line intensities, but no kinematical information. Recently, the SWAS and Odin satellites have provided measurements of the ortho- $\mathrm{H}_{2} \mathrm{O}$ ground-state line at $557 \mathrm{GHz}$ at high spectral resolution (Harwit \& Bergin 2002; Justtanont et al. 2005). Modelling of circumstellar $\mathrm{H}_{2} \mathrm{O}$ line emission is complicated by the very high optical depths, and the highly sub-thermal excitation in CSEs. The former leads to strong P Cygni profiles (when the continuum and line flux densities are comparable), which requires comparison with high-spectral-resolution data (e.g., the SWS lines of NML Cyg; Zubko et al. 2004). The latter leads to a dependence on collisional cross sections (where the ortho/para structures of both $\mathrm{H}_{2} \mathrm{O}$ and $\mathrm{H}_{2}$ produce an additional complication).

W Hya, a semiregular M-star, has been the target of a number of $\mathrm{H}_{2} \mathrm{O}$ investigations with highly disparate results (Barlow et al. 1996; Neufeld et al. 1996; Zubko \& Elitzur 2000). Recently, Justtanont et al. (2005) analysed Odin and ISO data. All available $\mathrm{H}_{2} \mathrm{O}$ line intensities could be fitted within the estimated observational uncertainties (Figure 4), and the mass-loss rate and the $\mathrm{H}_{2} \mathrm{O}$ abundance were both determined from the data (provided that the ISO SWS lines were included). The mass-loss rate is estimated to be about $2 \times 10^{-7} \mathrm{M}_{\odot} \mathrm{yr}^{-1}$, a result consistent with those obtained from CO radio line modelling and a fit to the SED combined with a dynamical model of a dust-driven 


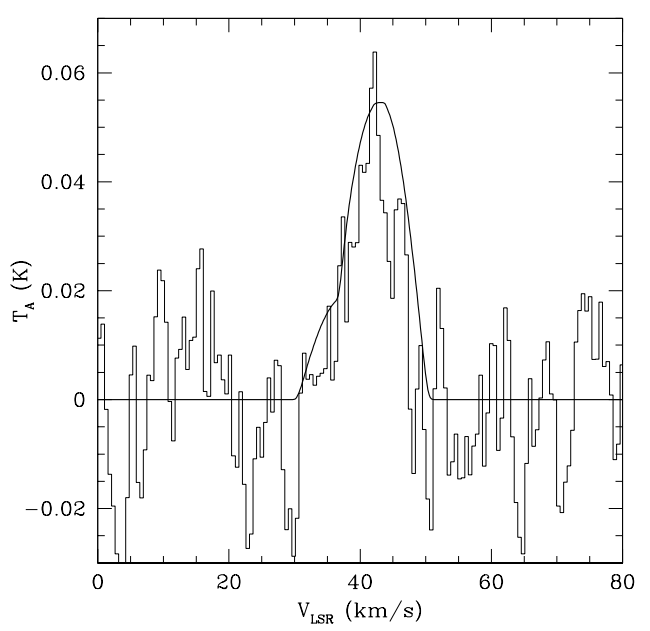

Figure 4. The $\mathrm{H}_{2} \mathrm{O}\left(1_{0,1}-1_{1,0}\right)$ line obtained towards the M-star W Hya by the Odin satellite, and a radiative transfer model fit. The line is strongly affected by self absorption due to a high optical depth.

wind. The resulting circumstellar $\mathrm{H}_{2} \mathrm{O}$ abundance (ortho + para) is $2 \times 10^{-3}$, i.e., about a factor of five higher than that expected from stellar atmosphere chemistry, and about a factor of two higher than allowed by solar abundances. This could reflect a problem with the radiative transfer modelling, e.g., optical depth effects, strong dependence on radiation fields (excitation to vibrational states was not included in the analysis), the use of erroneous collisional cross sections, or a too low mass-loss rate (although there are many indications that it is low). Nevertheless, an extra source of circumstellar $\mathrm{H}_{2} \mathrm{O}$ (with an associated underabundance of $\mathrm{H}_{2}$ ) remains an interesting possibility. Clearly, these results show that the interpretation of Herschel data on circumstellar $\mathrm{H}_{2} \mathrm{O}$ will require careful modelling and adequate input data.

\section{Summary}

Our understanding of circumstellar molecules is far from satisfactory due to the limited observational basis (the low number of sources observed, the lack of imaging data, etc.), and the limited use of detailed radiative transfer modelling. The results of the latter depend crucially on the properties of the circumstellar medium (morphology, density structure, radiation fields, chemistry, etc.), and basic molecular physics data since highly non-LTE excitation is the rule. The first studies of this type on larger samples suggest that very interesting results can be obtained.

Nevertheless, the extensive studies of the C-rich CSE of IRC+10216 have led to a number of secure results that can be compared in detail with astrochemical modelling predictions. It should be emphasized that we do not know to what extent these results are applicable to C-rich CSEs in general, i.e., the concept that the IRC+10216 CSE is the prototype of C-rich CSEs still rests on rather loose grounds. Further, comparisons of the IRC +10216 results with the extensive studies of the young C-rich post-AGB object AFGL618 strongly suggest that the increased UV flux and the presence of shocks make these objects efficient factories of more complex organic molecules.

Finally, even though this review has focussed on abundance determinations based on radio line observations, we can expect considerable progress when IR spectroscopy data 
will become available for a larger number of sources. These data contains such a wealth of lines that very good constraints can be put on the modelling, and, in addition, provide observations of non-polar molecules.

\section{References}

Apponi, A.J., McCarthy, M.C., Gottlieb, C.A., \& Thaddeus, P. 1999, Ap. J. 516, L103

Barlow, M.J., Nguyen-Q-Rieu, Truong-Bach, et al. 1996, A\&A 315, L241

Bujarrabal, V., Fuente, A., \& Omont, A. 1994, A\&A 285, 247

Cernicharo, J., Gottlieb, C.A., Guélin, M., et al. 1991, Ap. J. 368, L43

Cernicharo, J. \& Guélin, M. 1987, A\&A 183, L10

一. 1996, A\&A 309, L27

Cernicharo, J., Guélin, M., \& Kahane, C. 2000, A\&AS 142, 181

Cernicharo, J., Heras, A.M., Pardo, J.R., et al. 2001a, Ap. J. 546, L127

Cernicharo, J., Heras, A.M., Tielens, A.G.G.M., et al. 2001b, Ap. J. 546, L123

Cernicharo, J., Kahane, C., Guélin, M., \& Hein, H. 1987, A\& A 181, L9

Cox, P., Huggins, P.J., Maillard, J.-P., et al. 2002, A\&A 384, 603

Ford, K.E.S., Neufeld, D.A., Goldsmith, P.F., \& Melnick, G.J. 2003, Ap. J. 589, 430

Ford, K.E.S., Neufeld, D.A., Schilke, P., \& Melnick, G.J. 2004, Ap. J. 614, 990

González Delgado, D., Olofsson, H., Schwarz, H.E., et al. 2003, A\&\&A 411, 123

Groenewegen, M.A.T. 1994, A\&\&A 290, 531

Guélin, M., Neininger, N., \& Cernicharo, J. 1998, A\&3A 335, L1

Harwit, M. \& Bergin, E.A. 2002, Ap. J. 565, L105

Justtanont, K., Bergman, P., Larsson, B., et al. 2005, A\&A 439, 627

Kahane, C., Cernicharo, J., Gomez-Gonzalez, J., \& Guelin, M. 1992, A\&3A 256, 235

Kahane, C., Dufour, E., Busso, M., et al. 2000, A\& $A$ 357, 669

Latter, W.B., Walker, C.K., \& Maloney, P.R. 1993, Ap. J. 419, L97

Melnick, G.J., Neufeld, D.A., Ford, K.E.S., Hollenbach, D.J., \& Ashby, M.L.N. 2001, Nature 412,160

Millar, T.J. 2003, in H.J. Habing \& H.Olofsson (eds), Asymptotic giant branch stars, Astronomy and astrophysics library (New York, Berlin: Springer), p. 247

Neufeld, D.A., Chen, W., Melnick, G.J., et al. 1996, A\&A, 315, L237

Ohishi, M., Kaifu, N., Kawaguchi, K., et al. 1989, Ap. J. 345, L83

Olofsson, H. 2003, in H.J. Habing \& H.Olofsson (eds), Asymptotic giant branch stars, Astronomy and astrophysics library (New York, Berlin: Springer), p. 325

Olofsson, H., González Delgado, D., Kerschbaum, F., \& Schöier, F. 2002, A\&3A 391, 1053

Olofsson, H., Lindqvist, M., Winnberg, A., Nyman, L.-Å., \& Nguyen-Q-Rieu. 1991, A $\& A$ 245, 611

Omont, A., Lucas, R., Morris, M., \& Guilloteau, S. 1993, A\&SA 267, 490

Pardo, J.R., Cernicharo, J., \& Goicoechea, J.R. 2005, Ap. J. 628, 275

Schöier, F.L. \& Olofsson, H. 2000, A\&A 359, 586

—. 2001, A\&SA 368, 969

-. 2005, $A \mathscr{E} A$, submitted

Schöier, F.L., Olofsson, H., Wong, T., Lindqvist, M., \& Kerschbaum, F. 2004, A\&̛A 422, 651

Schöier, F.L., van der Tak, F.F.S., van Dishoeck, E.F., \& Black, J.H. 2005, A $\& A$ A 432, 369

Wannier, P.G., Andersson, B.-G., Olofsson, H., Ukita, N., \& Young, K. 1991, Ap. J. 380, 593

Woods, P.M., Millar, T.J., Herbst, E., \& Zijlstra, A.A. 2003a, A $\mathscr{S} A$ 402, 189

Woods, P.M., Schöier, F.L., Nyman, L.-Å., \& Olofsson, H. 2003b, A\&SA 402, 617

Ziurys, L.M., Savage, C., Highberger, J.L., et al. 2002, Ap. J. 564, L45

Zubko, V. \& Elitzur, M. 2000, Ap. J. 544, L137

Zubko, V., Li, D., Lim, T., Feuchtgruber, H., \& Harwit, M. 2004, Ap. J. 610, 427 


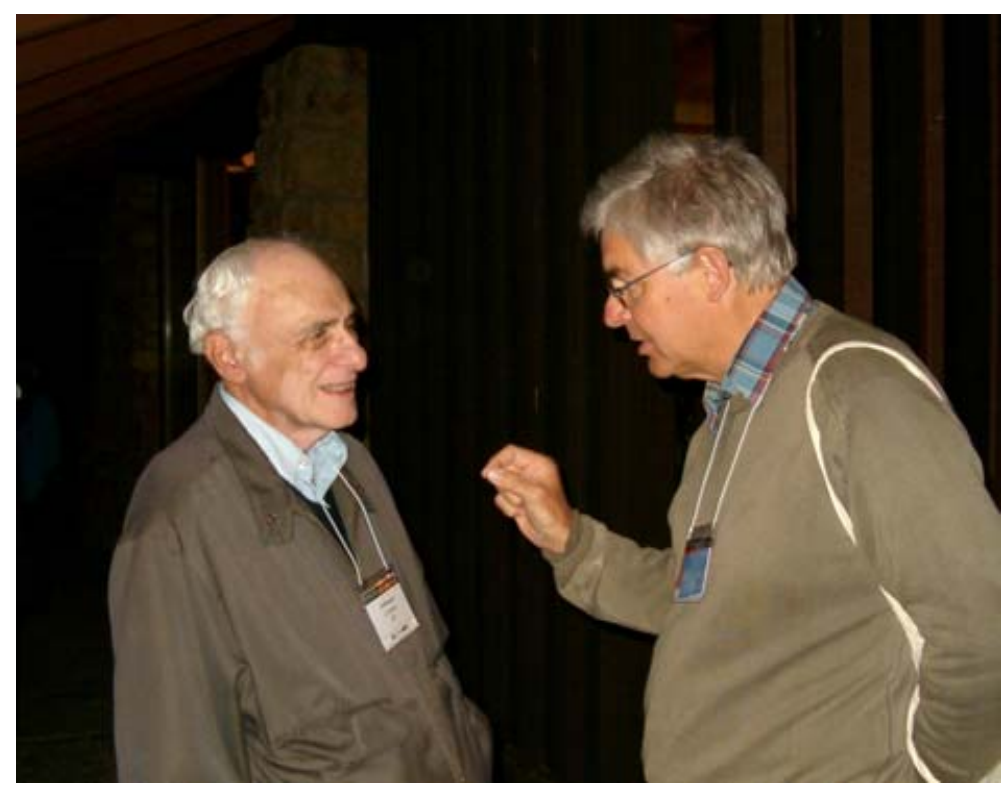

Photo: E. Herbst 